Tropical Journal of Pharmaceutical Research May 2021; 20 (5): 917-924

ISSN: $1596-5996$ (print); 1596-9827 (electronic)

(C) Pharmacotherapy Group, Faculty of Pharmacy, University of Benin, Benin City, 300001 Nigeria

Available online at http://www.tjpr.org

Original Research Article

http://dx.doi.org/10.4314/tjpr.v20i5.5

\title{
Rhotekin 2 promotes progression of endometrial carcinoma by activating Akt/GSK3 $\beta$ signaling pathway
}

\author{
Ting Hu${ }^{1}$, Min Wang ${ }^{1}$, Jing Zeng ${ }^{1}$, Xiaoli Wang ${ }^{1}$, Hangzhi Gu ${ }^{2 *}$ \\ ${ }^{1}$ Department of Obstetrics and Gynecology, Chengdu Second People's Hospital, Chengdu, Sichuan Province 610017, \\ ${ }^{2}$ Department of Gynecology, The First Affiliated Hospital of Wenzhou Medical University, Wenzhou, Zhejiang Province 325000, \\ China
}

*For correspondence: Email: guhangzhi2021@163.com; Tel: +86-0577-88069303

Sent for review: 8 February 2021

Revised accepted: 28 April 2021

\begin{abstract}
Purpose: To investigate the expression of rhotekin 2 (RTKN2) in human endometrial carcinoma tissues and its role in cancer progression.

Methods: Quantitative polymerase chain reaction ( $q P C R)$ and immunoblot assays were performed to determine the expression of RTKN2 in endometrial carcinoma tissues and cells. The effects of RTKN2 on cell proliferation were assessed using cell counting kit-8 and EdU assays, while its effects on cell cycle and apoptosis in endometrial carcinoma cells were evaluated by flow cytometry. The involvement of RTKN2 in activating Akt/glycogen synthase kinase-3 beta (GSK3 $\beta$ ) pathway was characterized using an immunoblot assay. Tumor growth assays were performed to assess the effects of RTKN2 on the progression of endometrial carcinoma in vivo, while protein expression in tumor tissues was determined by an immunohistochemical assay.

Results: High expression of RTKN2 was found in human endometrial carcinoma tissues and cell lines $(p<0.05)$. RTKN2 promoted the proliferation and cell cycle of endometrial carcinoma cells in vitro and suppressed apoptosis $(p<0.05)$. RTKN2 also activated Akt/GSK3 $\beta$ pathway in endometrial carcinoma cells and thus promoted tumor growth in vivo.

Conclusion: The involvement of RTKN2 in the progression of endometrial carcinoma has been confirmed in this study. RTKN2 is thus a promising therapeutic target for patients with this disease.
\end{abstract}

Keywords: Rhotekin 2 (RTKN2), Endometrial carcinoma, Proliferation, Apoptosis, Akt/GSK3 $\beta$

\begin{abstract}
This is an Open Access article that uses a fund-ing model which does not charge readers or their institutions for access and distributed under the terms of the Creative Commons Attribution License (http://creativecommons.org/licenses/by/4.0) and the Budapest Open Access Initiative (http://www.budapestopenaccessinitiative.org/read), which permit unrestricted use, distribution, and reproduction in any medium, provided the original work is properly credited.

Tropical Journal of Pharmaceutical Research is indexed by Science Citation Index (SciSearch), Scopus, International Pharmaceutical Abstract, Chemical Abstracts, Embase, Index Copernicus, EBSCO, African Index Medicus, JournalSeek, Journal Citation Reports/Science Edition, Directory of Open Access Journals (DOAJ), African Journal Online, Bioline International, Open-J-Gate and Pharmacy Abstracts
\end{abstract}

\section{INTRODUCTION}

Endometrial carcinoma (EC) is an epithelial malignancy [1]. With recent improvements in living standards, the rate of obesity in women continues to increase, resulting in an increase in the incidence of EC. Patients with early EC generally have a good prognosis. However, patients with advanced EC generally have a poor prognosis [2]. Although there are several treatments for this disease, including surgery, chemoradiotherapy, and targeted therapies, treatment options for patients with advanced EC remain limited [3]. To improve the prognosis of patients with EC, it is of vital importance to understand the pathogenesis of this disease and identify new therapeutic targets. 
Rhotekin 2 (RTKN2), first seen in the cytosol and nuclei of $\mathrm{CHO}$ cells, is a member of the rhotekin protein family [4]. RTKN1 and RTKN2 family members have both an $\mathrm{N}$-terminal HR1 domain and a pleckstrin homology domain and can bind Rho-GTPase [4]. Rho-GTPases are involved in the regulation of cell growth and cytoskeletal organization [5]. RTKN2 also plays an important role in these cellular processes [4].

The involvement of RTKN2 in the progression of multiple types of cancer has been reported [6]. RTKN2 affects the proliferation, migration, and apoptosis of several types of cancer, such as colon cancer, osteosarcoma, and lung cancer [7]. RTKN2 is highly expressed in bladder cancer tissues, and the depletion of RTKN2 has been shown to inhibit the growth of bladder cancer. In hepatocellular carcinoma ( $\mathrm{HCC}$ ), high expression levels of RTKN2 were shown to increase the expression of proliferating cell nuclear antigen (PCNA), promoting HCC [8]. However, the effects of RTKN2 on EC is still unclear.

Herein, the expression of RTKN2 in human EC tissues and cell lines was assessed. Furthermore, the effects of RTKN2 on the proliferation and apoptosis of EC cells and the regulatory mechanism involved were further investigated.

\section{EXPERIMENTAL}

\section{Bioinformatic analyses}

Gene Expression Profiling Interactive Analysis (GEPIA) was used to analyze RTKN2 expression in EC tissues from The Cancer Genome Atlas database.

\section{Antibodies and plasmids}

Anti-RTKN2 antibody $\quad[1: 200$ for immunohistochemistry (IHC), 1:1,000 for immunoblot analyses, ab183505, abcam], antiKi67 antibody (1:200 for IHC, 1:2,000 for immunoblot analyses, ab15580, abcam), anti-pAkt antibody (1:200 for IHC, 1:2,000 for immunoblot analyses, ab 38449, abcam), anti-pGSK3 $\beta$ antibody (1:200 for IHC, $1: 2,000$ for immunoblot analyses, ab75814, abcam), anti-Akt antibody (1:500, ab8805, abcam), anti-GSK3 $\beta$ antibody (1:500, ab93926, abcam), anti-cyclin D1 antibody (1:1,000, ab16663, abcam) anti-CDK4 antibody (1:500, ab108357, abcam), anti-CDC6 antibody (1:500, ab109315, abcam), anti-Bax antibody (1:500, ab32503, abcam), anti-Bcl-2 antibody (1:500, ab182858, abcam), and anti- $\beta$ actin antibody (1:3,000, ab8226, abcam).

The RTKN2-pcDNA3.1, shRTKN2\#1, and shRTKN2\#2 plasmids were constructed.

\section{IHC}

This study was approved according to the Guide for the Care and Use of Laboratory Animals and the World Medical Association Declaration of Helsinki [9]. The expression levels of proteins in tumor tissues were determined using IHC assays. The sections (5- $\mu \mathrm{m}$-thick) were fixed in $4 \%$ paraformaldehyde (PFA) for $30 \mathrm{~min}$ and subsequently blocked with $2 \%$ bovine serum albumin for $30 \mathrm{~min}$. The blocked sections were then first incubated with primary antibodies for 2 $\mathrm{h}$ and then with biotinylated secondary antibodies for 1.5 h. Finally, a chromogen-containing substrate was used to visualize protein levels.

\section{Cell culture and transfection}

Human endometrial epithelial cells (HEECs) and three types of endometrial cancer cells (HEC1B, AN3CA, and Ishikawa cells) were purchased from the ATCC and incubated in DMEM supplemented with $10 \%$ fetal bovine serum at 37 ${ }^{\circ} \mathrm{C}$ in $5 \% \mathrm{CO}_{2}$.

\section{Quantitative PCR assays}

Total RNA was extracted from EC cells using TRIzol reagent (15596-018, Invitrogen) and reverse-transcribed using an M-MLV reverse transcriptase kit (M1701, Promega). Quantitative PCR was then performed using the SYBR mixture (RR420A, Takara). The primers used are shown in Table 1. The expression levels of RTKN2 were normalized to those of GAPDH.

\section{Immunoblot analysis}

Cells were lysed in RIPA buffer (9800; Cell Signaling). The proteins were separated using SDS-PAGE,

Table 1: Primers used for quantitative PCR

\begin{tabular}{lll}
\hline Gene & Forward primers & Reverse primers \\
\hline RTKN2 & ATGCTCGACTAATGGCCTATACA & CGTCGTGATCGTTCTTTATTGCT \\
GAPDH & CGACCACTTTGTCAAGCTCA & GGTTGAGCACAGGGTACTTTATT \\
\hline
\end{tabular}


and then the proteins in the gels were transferred onto nitrocellulose (NC) membranes, followed by blocking of the membranes in TBST containing $5 \%$ fat-free milk. All NC membranes were subsequently incubated with primary antibodies for $1.5 \mathrm{~h}$ prior to incubation with secondary antibodies for $1 \mathrm{~h}$. Signals were then detected using an immunoblot exposure meter.

\section{CCK-8 assays}

CCK-8 assays were performed as described previously [10]. Briefly, cells were plated and subsequently maintained for $48 \mathrm{~h}$ with the indicated treatment. Cells were then treated with CCK-8 for $4 \mathrm{~h}$, and the OD values were measured at a wavelength of $490 \mathrm{~nm}$.

\section{EdU proliferation assays}

Cells were plated into 24-well plates at a density of 2,000 cells/well and subsequently maintained for $48 \mathrm{~h}$ with the indicated treatment. Cells were then treated with CCK-8 for $4 \mathrm{~h}$ followed by the addition of $50 \mu \mathrm{M}$ EdU. Immunofluorescence assays were then performed to determine the number of EdU-positive cells.

\section{Cell cycle assays}

Cells were fixed in $70 \%$ ethyl alcohol for $24 \mathrm{~h}$ at $-20{ }^{\circ} \mathrm{C}$ and incubated with $100 \mu \mathrm{g} / \mathrm{mL}$ propidium iodide (PI) at $37{ }^{\circ} \mathrm{C}$ for 20 min prior to analysis. The percentage of cells in each phase of cell cycle was analyzed by flow cytometry.

\section{Cell apoptosis assay}

Cells were resuspended in PBS, incubated with annexin V-FITC and PI for $10 \mathrm{~min}$, and subsequently analyzed using flow cytometry. Cells in different groups were analyzed and compared.

\section{In vivo evaluation of tumor growth}

All animal experiments were approved by the Ethics Committee of The First Affiliated Hospital of Wenzhou Medical University(Approval No. nyd002021-0003) and conducted in accordance with the National Institutes of Health Laboratory Animal Care and Use Guidelines [11]. BALB/C nude mice ( $20 \mathrm{~g}$, female, 6 weeks of age) were purchased from Beijing Vital River. All mice in this study were fed food and water ad libitum and housed in a specific pathogen-free facility. Ten nude mice were used in this study. To measure tumor growth capacity in vivo, HEC1B cells were stably transfected with RTKN2 shRNA plasmids and injected into mice. The tumor volume was measured every 7 days. After 28 days, tumor growth curves were plotted and compared between different groups.

\section{Statistical analyses}

GraphPad 6.0 was used for statistical analyses. Data are presented as the mean \pm standard error of the mean (SEM). The Student's $t$ test was used for data comparison in this study, and $P$ values less than 0.05 were considered statistically significant.

\section{RESULTS}

\section{RTKN2 is highly expressed in human EC} tissues and cell lines

To investigate the presence of RTKN2 in EC, RTKN2 expression levels were detected using GEPIA. Interestingly, we noticed that the expression of RTKN2 in 306 tumor tissues was significantly higher than in 13 normal tissues (Figure $1 \mathrm{~A}$ ). Quantitative PCR assays were performed to measure the levels of RTKN2 mRNA in human EC tissues and adjacent normal tissues that were collected when patients were hospitalized. Consistently, increased RTKN2 mRNA levels were found in EC tissues (Figure 1 B). Furthermore, using immunoblot assays, RTKN2 protein expression levels were detected in three representative EC tissues and adjacent normal tissues from hospitalized patients. As expected, protein levels of RTKN2 were higher in $\mathrm{EC}$ tissues than in normal tissues (Figure $1 \mathrm{C}$ ).

The mRNA and protein levels of RTKN2 were found in HEECs and three types of endometrial cancer cells, including HEC1B, AN3CA, and Ishikawa cells, using qPCR and immunoblot assays. The results confirmed higher expression levels of RTKN2 in HEC1B, AN3CA, and Ishikawa cells than in HEECs (Figures 1D, E). Therefore, RTKN2 was found to be highly expressed in human EC.

\section{RTKN2 promotes the proliferation of EC cells in vitro}

The effects of RTKN2 on EC were investigated. The RTKN2 overexpression plasmid RTKN2pcDNA3.1 and the shRNA plasmids shRTKN2\#1 and shRTKN2\#2 were transfected into two different EC cell lines (HEC1B and AN3CA) to study changes in protein expression. As seen in immunoblot assays, the transfection of the RTKN2-pcDNA3.1 plasmid increased RTKN2 expression in HEC1B and AN3CA cells (Figure 2 A). However, transfection of shRTKN2\#1 and shRTKN2\#2 led to decreased RTKN2 expression 
in HEC1B and AN3CA cells (Figure $2 \mathrm{~A}$ ). The overexpression of RTKN2 induced the proliferation of HEC1B and AN3CA cells (Figure 2 B), whereas the depletion of RTKN2 suppressed the viability of HEC1B and AN3CA cells, as observed by the decreased OD value (Figure 2 B). Also, using EdU assays, the overexpression of RTKN2 increased the number of EdU-positive HEC1B and AN3CA cells, whereas the depletion of RTKN2 decreased the number of EdU-positive HEC1B and AN3CA cells (Figure $2 \mathrm{C}$ ).
A

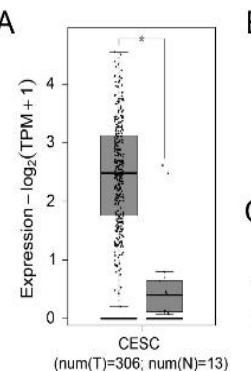

D

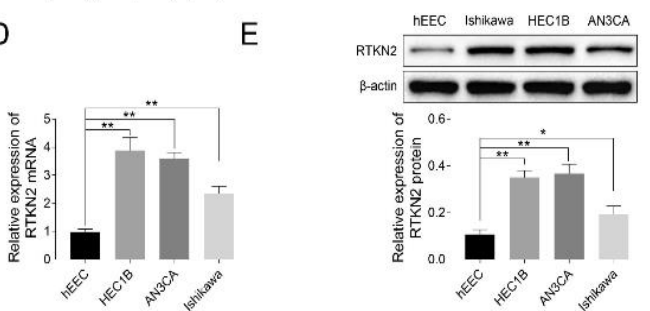

Figure 1: RTKN2 is upregulated in human EC tissues and cell lines. (A). GEPIA showing higher expression levels of RTKN2 in 306 tumor tissues than in 13 normal tissues. (B) Results of quantitative PCR assays showing RTKN2 mRNA levels in EC tissues and adjacent tissues. (C) Results of immunoblot assays showing expression of RTKN2 in three representative EC tissues and adjacent tissues. (D) Results of quantitative PCR assays showing RTKN2 expression in HEECs and three types of EC cells (HEC1B, AN3CA, and Ishikawa cells). $€$ Results of immunoblot assays showing the expression levels of RTKN2 in HEECs and in HEC1B, AN3CA, and Ishikawa cells. Data are presented as the mean $\pm \mathrm{SEM},{ }^{* *} P<0.01$, ${ }^{* * *} P<0.001$

\section{RTKN2 mediates the cell cycle of EC cells}

Since RTKN2 expression affected the proliferation of EC cells, whether RTKN2 could affect the cell cycle of EC UI cells was investigated. Interestingly, RTKN2 overexpression increased the percentages of HEC1B and AN3CA cells in the G1 and S phases, whereas its depletion decreased the percentages of HEC1B and AN3CA cells in the G1 phase (Figure $3 \mathrm{~A}$ ). The results of the immunoblot assays revealed that the overexpression of RTKN2 increased the expression levels of CDK4, cyclin D1, and CDC6, three cell cycle regulators (Figure $3 \mathrm{~B}$ ). RTKN2 depletion downregulated CDK4, cyclin D1, and CDC6 in both HEC1B and AN3CA cells (Figure 3 $B)$. Therefore, RTKN2 was shown to mediate cell cycle in EC cells.

\section{RTKN2 depletion stimulates the apoptosis of EC cells}

We then investigated whether RTKN2 could affect the apoptosis of EC cells. Caspase-3 activity was decreased after RTKN2 overexpression in HEC1B and AN3CA cells, suggesting the inhibition of apoptosis (Figure 4 A). The depletion of RTKN2 increased caspase-3 activity in HEC1B and AN3CA cells (Figure $4 \mathrm{~A}$ ). To confirm these findings, immunostaining was performed, and RTKN2 overexpression decreased the staining intensity of Bax in HEC1B and AN3CA cells, whereas RTKN2 depletion increased the staining intensity (Figure $4 \mathrm{~B}$ ). Conversely, overexpression of RTKN2 increased the staining intensity of Bcl-2, and RTKN2 knockdown decreased the staining intensity in HEC1B and AN3CA cells (Figure 4 C). Collectively, RTKN2 was shown to suppress the apoptosis of EC cells.
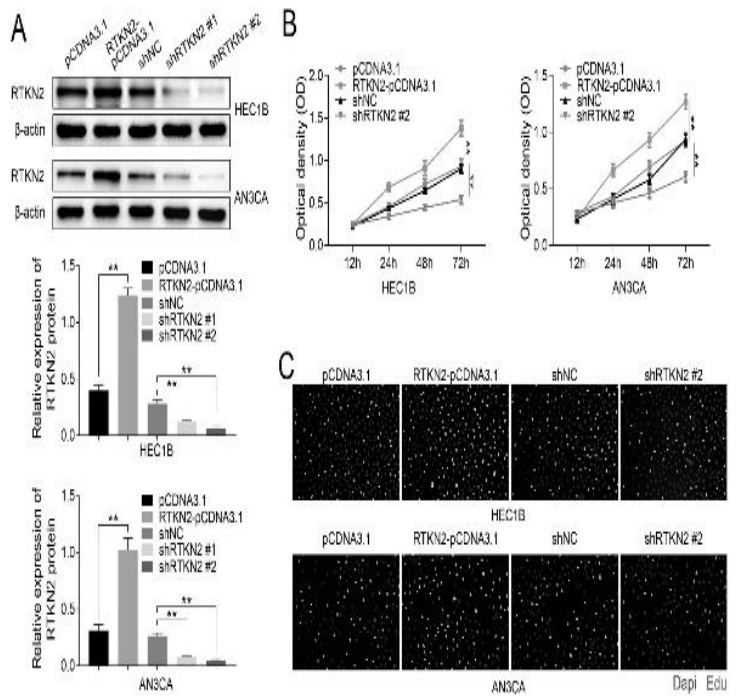

Figure 2: RTKN2 promotes the proliferation of EC cells in vitro. (A) Results of the immunoblot analysis showing the expression of RTKN2 in HEC1B and AN3CA cells transfected with the indicated plasmids. (B) CCK-8 assay results showing the proliferative capacity HEC1B and AN3CA cells transfected with the indicated plasmids. (C) Results of the EdU assays showing EdU-positive HEC1B and AN3CA cells transfected with the indicated plasmids. Data are presented as the mean \pm SEM; ${ }^{* \star} P<0.01$

Trop J Pharm Res, May 2021; 20(5): 920 


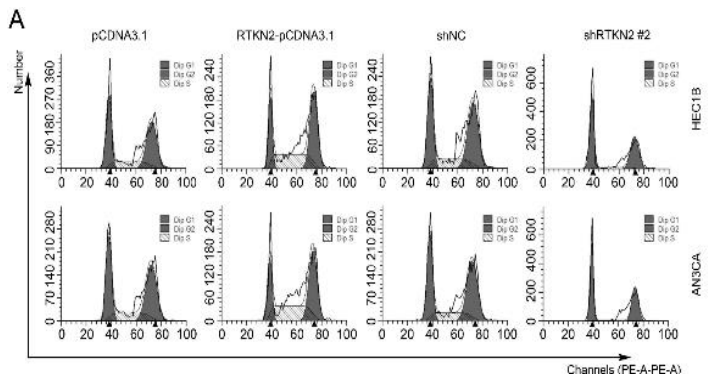

activate the Akt/GSK3 $\beta$ signaling pathway in EC cells.

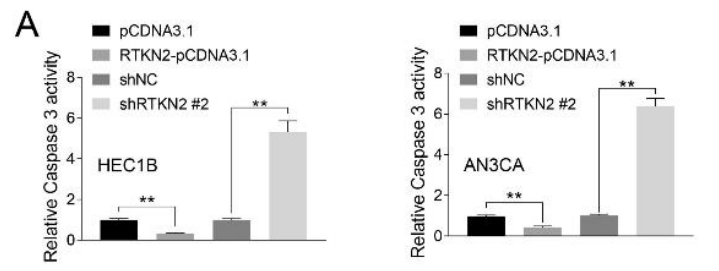

B
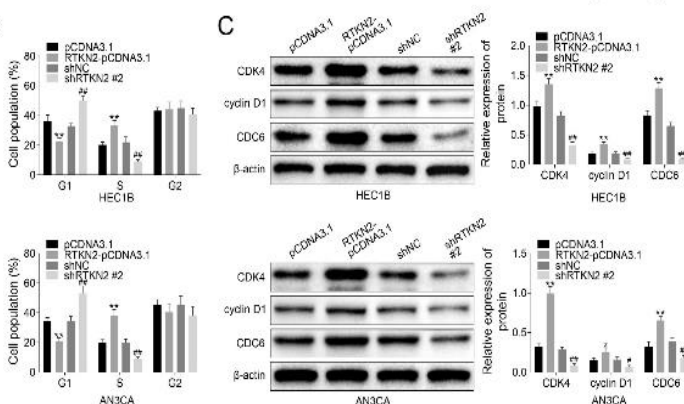

Figure 3: RTKN2 mediates the cell cycle of EC cells. (A) Flow cytometric analyses were performed to identify the number of HEC1B and AN3CA cells transfected with the indicated plasmids in different phases of the cell cycle. The percentages of cells in the $\mathrm{G} 1, \mathrm{~S}$, and $\mathrm{G} 2 / \mathrm{M}$ phases were quantified. (B) Results of the immunoblot analyses showing the expression levels of CDK4, cyclin D1, and CDC6 in HEC1B and AN3CA cells transfected with the indicated plasmids. NC, negative control, shNC, sh negative control. Data are presented as the mean \pm SEM, pcDNA3.1-RTKN2 vs. pcDNA3.1, ${ }^{*} P<0.05,{ }^{*} P$ $<0.01$, shRTKN2 vs. shNC, \#P<0.05, \#\#P<0.01

\section{RTKN2 activates the Akt/ glycogen synthase kinase-3 beta pathway in EC cells}

Several studies reported that the Akt/glycogen synthase kinase-3 beta (GSK3 $\beta$ ) signaling pathway was widely involved in the regulation of cancer cell proliferation and apoptosis [12]. The involvement of RTKN2 in promoting EC cell proliferation and suppressing apoptosis via this pathway was investigated.

Based on the immunoblot data, RTKN2 overexpression increased the phosphorylation of AKT and GSK3B(Ser9) in both HEC1B and AN3CA cells (Figure 5). The depletion of RTKN2 reduced the phosphorylation of AKT and GSK3 in both HEC1B and AN3CA cells (Figure 5), confirming the regulation of this pathway by RTKN2. RTKN2-depleted HEC1B and AN3CA cells were treated with perifosine, an inhibitor of the Akt pathway, and the effects of the treatment were measured. Perifosine treatment further suppressed the Akt/GSK3 $\beta$ pathway after RTKN2 depletion in HEC1B and AN3CA cells (Figure 5). Therefore, RTKN2 was shown to
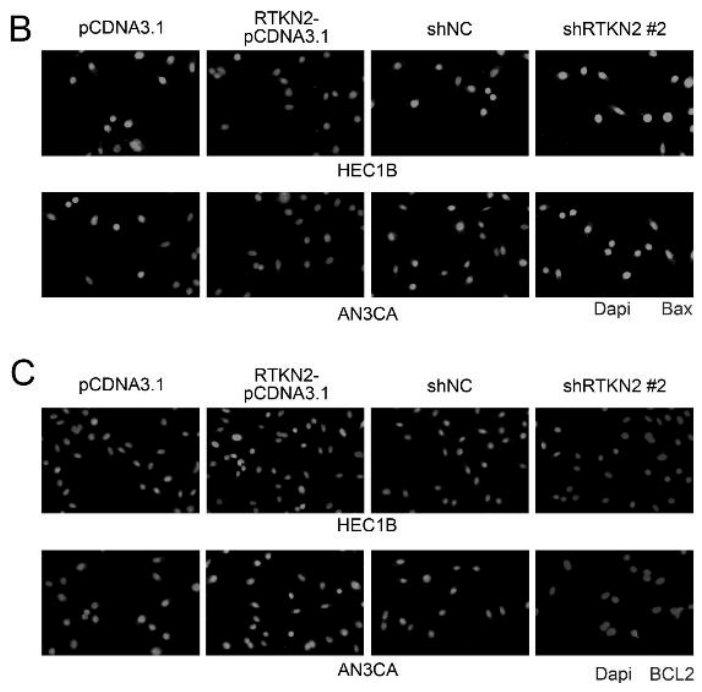

Figure 4: RTKN2 depletion stimulates the apoptosis of EC cells. (A) Caspase- 3 activity was measured in HEC1B and AN3CA cells after transfection with the indicated plasmids. (B) Immunofluorescence results showing Bax expression in HEC1B and AN3CA cells with the indicated treatment. (C) Immunofluorescence results showing $\mathrm{Bcl}-2$ expression in HEC1B and AN3CA cells with the indicated treatment. Data are presented as the mean \pm SEM, ${ }^{* *} P<0.01$

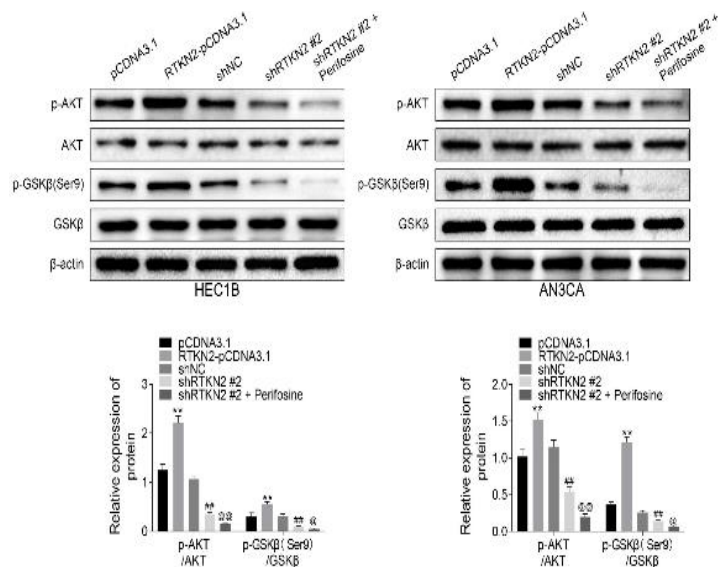

Figure 5: RTKN2 activates the Akt/GSK3 $\beta$ signaling pathway in EC cells. Results of the immunoblot analysis showing the expression of Akt, GSK3 $\beta$, and the phosphorylated forms of Akt and GSK3 $\beta$ in HEC1B and AN3CA cells transfected with the indicated plasmids and treated with the indicated drugs. Quantification 
was performed. Data are presented as the mean \pm SEM, pcDNA3.1-RTKN2 vs. pcDNA3.1, ${ }^{*} P<$ $0.05,{ }^{* *} P<0.01$, shRTKN2 vs. shNC, \#P<0.05, \#\#P $<0.01$. NC, negative control, shNC, sh negative control. shRTKN2+perifosine vs. shRTKN2, @P $<0.05, @ @ P<0.01$

RTKN2 promotes the growth of EC cellbased tumors in mice

The effects of RTKN2 on the growth of EC cellbased tumors were examined in vivo. HEC1B cells were stably transfected with control, shNC, or shRTKN2\#2 plasmids. A total of $10^{5}$ cells were then injected subcutaneously into the abdomens of nude mice. After 7 days, tumors began forming. The tumor volume was measured every 7 days for 28 days. Interestingly, RTKN2 depletion suppressed the growth of EC cellbased tumors (Figure $6 \mathrm{~A}$ ). The tumor weights decreased significantly in RTKN2 knockdown groups (Figure $6 \mathrm{~A}$ ). Using TUNEL assays, RTKN2 depletion in tumor tissues was shown to promote the apoptosis of EC cells, consistent with previous results (Figure $6 \mathrm{~B}$ ). As shown by $\mathrm{IHC}$ assays, the depletion of RTKN2 decreased the expression levels of Ki67 and RTKN2, as well as the phosphorylation of Akt and GSK3 $\beta$, in tumor tissues, consistent with previous data (Figure $6 \mathrm{C}$ ). Therefore, RTKN2 was shown to promote the growth of EC cell-based tumors in vivo.

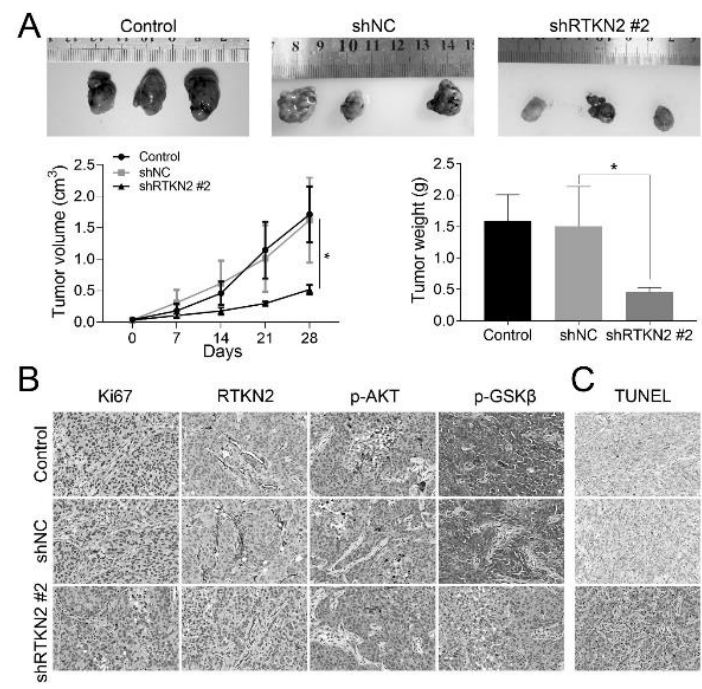

Figure 6: RTKN2 promotes the growth of EC cellbased tumors in mice. (A) Control (NC), shNC, and RTKN2 shRNA plasmids were stably transfected into HEC1B cells, and the cells were then injected subcutaneously into the abdomens of nude mice to induce tumor growth. The tumor growth curves were plotted according to tumor volume measurements (every 7 days), and tumor weights were measured. (B) TUNEL assays were performed to detect the degree of apoptosis in tumor tissues from mice in the NC, shNC, and shRTKN2 groups. (C). IHC assays were performed to assess the expression levels of Ki67, RTKN2, phosphorylated Akt, and phosphorylated GSK3 $\beta$ in tumor tissues from mice in the NC, shNC, and shRTKN2 groups. Data are presented as the mean \pm SEM, ${ }^{*} P<$ 0.05

\section{DISCUSSION}

EC is an epithelial cell malignancy of the endometrium, with nearly 200,000 new cases diagnosed every year. It is the most common cancer of the female reproductive tract in the United States, and its incidence has also been increasing annually in China [13]. Although early EC has a high cure rate, once this cancer has reached an advanced stage, existing treatment methods, such as surgical resection, radiotherapy, and chemotherapy, have little impact on patient prognosis. In recent years, targeted therapy for EC has attracted a lot of attention; many new therapeutic targets have been developed, and a few targeted therapies are in clinical trials with promising results [1].

To further improve patient outcomes, new and more effective therapeutic targets need to be developed. Consistent with previous studies reporting high mRNA levels of RTKN2 in human EC tissues, high expression of RTKN2 in EC tissues and cell lines was confirmed using bioinformatics analyses, quantitative PCR, and immunoblot assays. The data suggest that RTKN2 is a promising molecular target for the treatment of EC.

RTKN2, a Rho-GTPase binding protein, mediates multiple cellular processes. Interestingly, in this study, the depletion of RTKN2 suppressed the proliferation of EC cells and stimulated cell cycle arrest, suggesting that RTKN2 affects Rho-GTPase-related cellular processes. The suppression of EC cell apoptosis by RTKN2 was also observed.

RTKN2 has been demonstrated to regulate the proliferation, migration, apoptosis, and cell cycle of multiple types of cancer [14]. Similar to effects seen in osteosarcoma, RTKN2 depletion led to cell cycle arrest. A microRNA, miR-181, was reported to regulate the nuclear factor kappa $B$ pathway by targeting RTKN2 in ovarian cancer [15]. In this study, RTKN2 contributed to the progression of EC via the Akt/GSK3 $\beta$ pathway, suggesting the presence of multiple regulatory effects of RTKN2 on tumorigenesis. In HCC cells, increasing the expression of RTKN2 led to the upregulation of PCNA, promoting cellular proliferation and metastasis [8]. 
Another study showed that phosphorylated PCNA activated the ATM/Akt/GSK3 $\beta / S n a i l$ axis, thereby increasing the proliferation and migratory ability of tumor cells. Whether RTKN2 contributes to the progression of EC requires further study. In fact, the Akt/GSK3 $\beta$ axis is widely involved in the regulation of cancer cell proliferation and apoptosis in multiple types of cancer, such as breast cancer and lung cancer, consistent with the findings of this study. This pathway could also serve as a therapeutic target for the treatment of EC.

\section{CONCLUSION}

High RTKN2 expression was found in human EC tissues and cell lines. RTKN2 promoted the proliferation of EC cells by regulating the cell cycle and suppressing apoptosis. RTKN2 protein activated the Akt/GSK3 $\beta$ pathway and thereby promoted the progression of EC. RTKN2 protein is therefore a potential molecular target for the treatment of EC.

\section{DECLARATIONS}

\section{Acknowledgement}

This work was supported by Chengdu Medical Research Project (Grant no. 2016011).

\section{Competing interest}

There are no conflicts of interest to disclose.

\section{Contribution of authors}

We declare that this work was done by the authors named in this article, and all liabilities pertaining to claims related to the content of this article will be borne by the authors. Ting $\mathrm{Hu}$ and Min Wang designed the study and supervised the data collection. Jing Zeng and Xiaoli Wang analyzed the data and interpreted the data. Hangzhi $\mathrm{Gu}$ prepared the manuscript for publication and reviewed the draft of the manuscript. All authors have read and approved the manuscript.

\section{Open Access}

This is an Open Access article that uses a funding model which does not charge readers or their institutions for access and distributed under the terms of the Creative Commons Attribution License (http://creativecommons.org/licenses/by/ 4.0) and the Budapest Open Access Initiative (http://www.budapestopenaccessinitiative.org/rea d), which permit unrestricted use, distribution, and reproduction in any medium, provided the original work is properly credited.

\section{REFERENCES}

1. Dou Y, Kawaler EA, Cui Zhou D, Gritsenko MA, Huang C, Blumenberg L, Karpova A, Petyuk VA, Savage SR, Satpathy $S$ et al. Proteogenomic Characterization of Endometrial Carcinoma. Cell 2020; 180(4): 729-748 e726.

2. Li Y, Huo J, He J, Zhang Y, Ma X. BTG1 inhibits malignancy as a novel prognosis signature in endometrial carcinoma. Cancer Cell Int 2020; 20: 490.

3. Wadee $R$, Grayson W. A potpourri of pathogenetic pathways in endometrial carcinoma with a focus on Lynch Syndrome. Ann Diagn Pathol 2019; 39: 92-104.

4. Ito $H$, Morishita $R$, Nagata Kl. Functions of Rhotekin, an Effector of Rho GTPase, and Its Binding Partners in Mammals. Int J Mol Sci 2018; 19(7).

5. Nagata $K$, Ito $H$, Iwamoto I, Morishita $R$, Asano $T$. Interaction of a multi-domain adaptor protein, vinexin, with a Rho-effector, Rhotekin. Med Mol Morphol 2009; 42(1): 9-15.

6. Pang X, Li R, Shi D, Pan X, Ma C, Zhang G, Mu C, Chen W. Knockdown of Rhotekin 2 expression suppresses proliferation and induces apoptosis in colon cancer cells. Oncol Lett 2017; 14(6): 8028-8034.

7. Ji L, Huang Y, Zhang Y, Peng A, Qin J, Lu S, Huang Y. RTKN2 is Associated with Unfavorable Prognosis and Promotes Progression in Non-Small-Cell Lung Cancer. Onco Targets Ther 2020; 13: 10729-10738.

8. Wei W, Chen H, Liu S. Knockdown of Rhotekin 2 expression suppresses proliferation and invasion and induces apoptosis in hepatocellular carcinoma cells. Mol Med Rep 2016; 13(6): 4865-4871.

9. Liao YX, Zeng JM, Zhou JJ, Yang GH, Ding $K$, Zhang XJ. Silencing of RTKN2 by siRNA suppresses proliferation, and induces $\mathrm{G1}$ arrest and apoptosis in human bladder cancer cells. Mol Med Rep 2016; 13(6): 4872-4878.

10. Gang L, Dong-Gang Z, Lai J, Yu-Hua G, Hua T, Gang Z, Xin $H$, Yan W. MiR-1254 inhibits proliferation, migration and invasion of human brain tumour cell lines. Trop $J$ Pharm Res 2018; 17(1): 35.

11. Care IoLARCo, Animals UoL: Guide for the care and use of laboratory animals: US Department of Health and Human Services, Public Health Service, National, 1986.

12. Xu JC, Chen TY, Liao LT, Chen T, Li QL, XU JX, Hu JW, Zhou $P H$, Zhang YQ. NETO2 promotes esophageal cancer progression by inducing proliferation and metastasis via PI3K/AKT and ERK pathway. Int J Biol Sci 2021; 17(1): 259-270.

13. Yu L, Wang S, Lin X, Lu Y, Gu P. MicroRNA-124a inhibits cell proliferation and migration in liver cancer by regulating interleukin-11. Mol Med Rep 2018; 17(3): 3972-3978. 
14. Wang $X$, Zhang $L$, Wang $W$, Wang $Y$, Chen $Y$, Xie $R$, Li $X$, Wang $Y$. Rhotekin 2 silencing inhibits proliferation and induces apoptosis in human osteosarcoma cells. Biosci Rep 2018; 38(6).
15. Lin Z, Li D, Cheng W, Wu J, Wang K, Hu Y. MicroRNA181 Functions as an Antioncogene and Mediates NFkappaB Pathway by Targeting RTKN2 in Ovarian Cancers. Reprod Sci 2019; 26(8): 1071-1081. 